\title{
BILIPTYSIS CAUSED BY A BRONCHOBILIARY FISTULA
}

Michael Poullis, Bsc(Hons), MBBS, FRCS(Eng), ${ }^{\mathrm{a}}$ and Andrew Poullis, Bsc(Hons), MBBS, MRCP, ${ }^{\mathrm{b}}$ London, United Kingdom

Bronchobiliary fistula is a rare condition that thoracic surgeons may be expected to encounter. It can be congenital or acquired (resulting from a biliary infection, ${ }^{1}$ malignancy, ${ }^{2}$ surgery, ${ }^{2,3}$ or trauma ${ }^{4}$ ). Surgery remains the mainstay of treat-

From the Department of Cardiothoracic Surgery, National Heart and Lung Institute, Imperial College of Science, Technology and Medicine, Hammersmith Hospital, ${ }^{\mathrm{a}}$ and the Department of Gastroenterology, Chelsea and Westminster Hospital, ${ }^{\mathrm{b}}$ London, United Kingdom.

Received for publication June 30, 1999; accepted for publication July 20, 1999.

Address for reprints: Michael Poullis, Bsc(Hons), MBBS, FRCS(Eng), British Heart Foundation Cardiothoracic Research Fellow, Department of Cardiothoracic Surgery, Hammersmith Hospital, Du Cane Rd, East Acton, London, W12 0NN, United Kingdom (E-mail: mpoullis@ rpms.ac.uk).

J Thorac Cardiovasc Surg 1999;118:971-2

Copyright $\odot 1999$ by Mosby, Inc.

$0022-5223 / 99 \$ 8.00+0 \quad \mathbf{1 2 / 5 4 / 1 0 1 5 5 7}$ ment of congenital bronchobiliary fistula. However, interventional radiology and gastroenterologic techniques of stenting provide a realistic alternative to surgical intervention in acquired fistula in patients, many of whom have significant concomitant medical conditions. 5

A 46-year-old woman with advanced colon carcinoma had a 10-day history of increasing jaundice. Ultrasonography revealed hilar lymphadenopathy and dilated intrahepatic ducts. An initial percutaneous ultrasound and fluoroscopically guided percutaneous transhepatic cholangiogram (PTC) revealed occlusion of the common hepatic duct but no bronchobiliary fistula. An external biliary stent was placed to decompress the biliary tree before elective biliary stenting.

Within the next 24 hours a productive cough developed with frank biliptysis. A bronchoscopic study demonstrated purulent bile-stained material throughout the bronchial tree, especially in the right lower lobe, but no obvious fistulous connection. Owing to the clinical diagnosis of bronchobiliary fistula, a biliary excretion scintigraphic (hepato-iminodiacetic acid, HIDA) scan was performed (Fig 1). This scan 

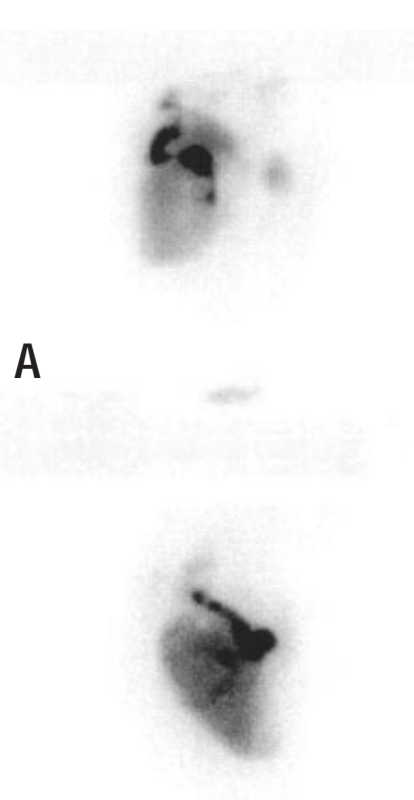

B

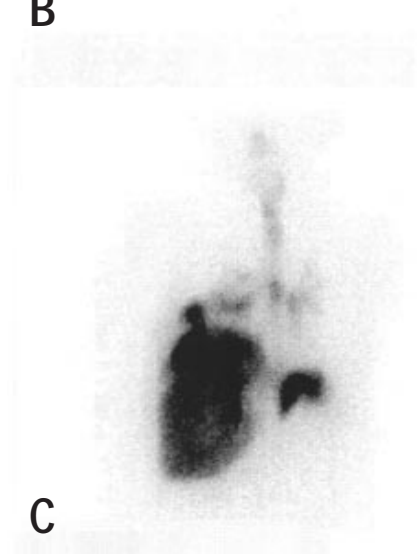

Fig 1. The right lobe of the liver shows good uptake of tracer (A). A focus appears on the right lateral surface of the liver (B), extends upward toward the chest, and can be seen widely in the chest including the trachea and mouth (C), confirming the diagnosis of bronchobiliary fistula.

revealed contrast medium in the biliary tree, which had a direct communication with the bronchial tree. Neither right lower lobectomy nor hepatic surgery was considered appro- priate because of the patient's condition. An expandable metal stent was deployed to relieve the biliary obstruction with the aid of a guide wire placed via the external biliary stent, which had been placed previously. Stenting resulted in a rapid improvement in the patient's clinical condition. Unfortunately, she died 9 months later of advanced colon carcinoma with no recurrence of the biliptysis.

This case demonstrates 4 important points. First, percutaneous hepatic interventions, which are very common in interventional radiologic units, can cross the pleural cavity with or without damaging the lung and can predispose a patient to the development of a bronchobiliary, pleurobiliary, or bronchopleural fistula. Second, although the literature emphasizes the surgical management of bronchobiliary fistulas, the surgical procedures, many of which are reoperative, are associated with significant morbidity and mortality. Third, HIDA scanning is a sensitive method of demonstrating bronchobiliary fistulas. Endoscopic retrograde cholangiopancreatography, PTC, and bronchoscopy are falling from favor as diagnostic methods if this condition is suspected. Fourth, biliptysis is the sine qua non of a bronchobiliary fistula.

Radiologic and gastrointestinal interventions via external and internal stenting, respectively, can be successful in reducing biliary obstruction. This conforms to the first point in the traditional 3-step surgical management of fistulas: remove distal obstruction, excision, or decrease flow. Only after nonoperative, interventional techniques have failed should operative approaches be considered, especially in patients with advanced concomitant diseases.

\section{REFERENCES}

1. Senturk H, Mert A, Ersavasti G, Tabak F, Akdogan M, Ulualp K. Bronchobiliary fistula due to alveolar hydatid disease: report of three cases. Am J Gastroenterol 1998;93:2248-53.

2. George TK, Carignan JR. Bronchobiliary fistula after hepatic resection for metastatic colon cancer. J Surg Oncol 1984;25:198200.

3. Rose DM, Rose AT, Chapman WC, Wright JK, Lopez RR, Pinson $\mathrm{CW}$. Management of bronchobiliary fistula as a late complication of hepatic resection. Am Surg 1998;64:873-6.

4. Coselli JS, Mattox KL. Traumatic bronchobiliary fistula. J Trauma 1983;23:161-2.

5. Brem H, Gibbons GD, Cobb G, Edgin RA, Ellison EC, Carey LC. The use of endoscopy to treat bronchobiliary fistula caused by choledocholithiasis. Gastroenterology 1990;98:490-2. 